\title{
IRREGULAR SETS ARE RESIDUAL
}

\author{
LUis BARREIRA, JinJun Li AND Claudia VAlls
}

(Received April 22, 2013, revised September 6, 2013)

\begin{abstract}
For shifts with weak specification, we show that the set of points for which the Birkhoff averages of a continuous function diverge is residual. This includes topologically transitive topological Markov chains, sofic shifts and more generally shifts with specification. In addition, we show that the set of points for which the Birkhoff averages of a continuous function have a prescribed set of accumulation points is also residual. The proof consists of bridging together strings of sufficiently large length corresponding to a dense set of limits of Birkhoff averages. Finally, we consider intersections of finitely many irregular sets and show that they are again residual. As an application, we show that the set of points for which the Lyapunov exponents on a conformal repeller are not limits is residual.
\end{abstract}

1. Introduction. Our main aim is to show that the irregular set of points for which the Birkhoff averages of a given continuous function diverge can be very large from the topological point of view. Namely, for shifts with weak specification we show that the irregular set is either empty or residual, even though in the context of ergodic theory it has zero measure with respect to any invariant measure. This includes topologically transitive topological Markov chains, sofic shifts and more generally shifts with specification, which means that any two strings can be bridged by a string of fixed length. As an application, we obtain a corresponding result for the Lyapunov exponents on a conformal repeller for a $C^{1}$ expanding map. Namely, we show that the set of points for which the Lyapunov exponents are not limits is a residual subset.

More precisely, let $f: X \rightarrow X$ be a continuous map on a compact metric space and let $\varphi: X \rightarrow \boldsymbol{R}$ be a continuous function. The irregular set for $\varphi$ is defined by

$$
X_{\varphi}=\left\{x \in X ; \liminf _{n \rightarrow \infty} \frac{1}{n} \sum_{i=0}^{n-1} \varphi\left(f^{i}(x)\right)<\limsup _{n \rightarrow \infty} \frac{1}{n} \sum_{i=0}^{n-1} \varphi\left(f^{i}(x)\right)\right\} .
$$

As a consequence of Birkhoff's ergodic theorem, the irregular set has zero measure with respect to any finite $f$-invariant measure on $X$. Therefore, at least from the point of view of ergodic theory, the set $X_{\varphi}$ can be discarded.

Remarkably, from the point of view of dimension theory the set $X_{\varphi}$ can be as large as the whole space. In particular, it was shown by Barreira and Schmeling in [6] that for a repeller $X$ of a $C^{1+\varepsilon}$ map $f$ that is topologically mixing and conformal on $X$ (the derivative of $f$ is a multiple of an isometry at each point of $X$ ), if $\varphi$ is Hölder continuous and is not cohomologous

2010 Mathematics Subject Classification. Primary 37B10.

Key words and phrases. Irregular sets, residual sets, topological Markov chains. 
to a constant then $X_{\varphi}$ is as large as the whole space from the points of view of topological entropy and Hausdorff dimension, that is,

$$
h\left(f \mid X_{\varphi}\right)=h(f \mid X) \text { and } \operatorname{dim}_{H} X_{\varphi}=\operatorname{dim}_{H} X,
$$

where $h(f \mid Z)$ is the topological entropy of $f$ on the set $Z \subset X$ and $\operatorname{dim}_{H} Z$ is the Hausdorff dimension of $Z$. We recall that $\varphi$ is said to be cohomologous to a constant if there exist a bounded function $\psi$ and a constant $c$ such that

$$
\varphi=\psi-\psi \circ f+c \text { on } X .
$$

Clearly, if $\varphi$ is cohomologous to a constant, then $X_{\varphi}$ is the empty set.

Now let $Y=\bigcup_{\varphi} X_{\varphi}$ be the union over all Hölder continuous functions. Under the former hypotheses, for a repeller $X$ we have

$$
h(f \mid Y)=h(f \mid X) \text { and } \operatorname{dim}_{H} Y=\operatorname{dim}_{H} X .
$$

The first identity in (2) was first established by Pesin and Pitskel in [13] for the full shift on two symbols. In a related direction, Shereshevsky [14] proved that for a generic $C^{2}$ surface diffeomorphism with a locally maximal hyperbolic set $X$ and an equilibrium measure $\mu$ of a Hölder continuous $C^{0}$-generic function, the set of points for which the pointwise dimension does not exist has positive Hausdorff dimension, that is,

$$
\operatorname{dim}_{H}\left\{x \in X ; \liminf _{r \rightarrow 0} \frac{\log \mu(B(x, r))}{\log r}<\limsup _{r \rightarrow 0} \frac{\log \mu(B(x, r))}{\log r}\right\}>0 .
$$

The identities in (1) also hold for topologically mixing topological Markov chains, although in this case the two are equivalent since up to a multiplicative constant the topological entropy and the Hausdorff dimension coincide. Again for topological Markov chains, the first identity in (1) was extended by Fan, Feng and Wu in [10] to arbitrary continuous functions. For repellers of $C^{1+\varepsilon}$ conformal maps, the second identity was extended by Feng, Lau and $\mathrm{Wu}$ in [11] to arbitrary continuous functions. We refer the reader to the book [2] for a detailed discussion of some of these results and to $[4,8,12,15]$ for further related work also for multifractal flows and maps with specification. In addition to showing that the irregular is large from the points of view of topological entropy and Hausdorff dimension, the topological pressure is also considered.

In another direction, it was noted in [6] that, under the former assumptions, the irregular set $X_{\varphi}$ is dense (this follows easily from the definitions). In the present paper we show, for shifts with specification and for an arbitrary continuous function $\varphi$, that the set $X_{\varphi}$ is residual (that is, $X_{\varphi}$ contains a dense $G_{\delta}$ set). This shows once more, in strong contrast to what happens in the context of ergodic theory, that the irregular set may be as large as the whole space from various points of view. Roughly speaking, the proof consists of bridging together strings of sufficiently large length corresponding to limits of Birkhoff averages. Some special irregular sets related to number theoretical properties of integer base representations were earlier shown to be residual in $[1,5]$. 
In fact, we study also a refined version of the irregular set. Namely, given an interval $I \subset \boldsymbol{R}$, let

$$
X_{I}=\left\{x \in X ; A_{\varphi}(x)=I\right\},
$$

where $A_{\varphi}(x)$ is the set of accumulation points of the sequence

$$
S_{\varphi}(x, n)=\frac{1}{n} \sum_{i=0}^{n-1} \varphi\left(f^{i}(x)\right) .
$$

When $I$ is not a singleton we show, for shifts with specification and for an arbitrary continuous function $\varphi$, that the set $X_{I}$ is also residual. We note that it is a subset of the irregular set $X_{\varphi}$ (when $I$ is not a singleton). Incidentally, $\mathrm{Li}$ and $\mathrm{Wu}$ studied in [12] the topological entropy of $X_{I}$ for a map $f$ with the specification property.

As an application of these results, we obtain corresponding statements for the averages $S_{\varphi}(x, n)$ when $X$ is a repeller for a $C^{1}$ expanding map $f$. More precisely, we show that when $\varphi: X \rightarrow \boldsymbol{R}$ is a continuous function and $I$ is not a singleton, then the set $X_{I}$ in (3) is residual (see Theorem 5.1). In particular, when $f$ is conformal on $X$ (which means that $d_{x} f$ is a multiple of an isometry for every $x \in X$ ) this implies that the Lyapunov exponent

$$
\lambda(x)=\limsup _{n \rightarrow \infty} \frac{1}{n} \log \left\|d_{x} f^{n}\right\|
$$

is not a limit on a residual subset. In other words, the set

$$
\left\{x \in X ; \liminf _{n \rightarrow \infty} \frac{1}{n} \log \left\|d_{x} f^{n}\right\|<\limsup _{n \rightarrow \infty} \frac{1}{n} \log \left\|d_{x} f^{n}\right\|\right\}
$$

is either empty or residual. This follows readily from the fact that since $f$ is conformal on $X$, we have

$$
\lambda(x)=\limsup _{n \rightarrow \infty} S_{\varphi}(x, n)
$$

taking $\varphi=\log \left\|d_{x} f\right\|$ (since $f$ is of class $C^{1}$, the function $\varphi$ is continuous). A related result can be obtained for the local entropy $h_{\mu}(x)$ of a Gibbs measure. We recall that if $\mu$ is a Gibbs measure of a continuous function $\psi$, assumed without loss of generality to have zero topological pressure, then

$$
h_{\mu}(x)=\lim _{n \rightarrow \infty}-\frac{1}{n} \log \mu\left(B_{n}(x, \varepsilon)\right)=\lim _{n \rightarrow \infty} S_{-\psi}(x, n),
$$

where

$$
B_{n}(x, \varepsilon)=\bigcap_{k=0}^{n-1} f^{-k} B\left(f^{k}(x), \varepsilon\right)
$$

(whenever both limits exist, in which case they are equal, where $\varepsilon$ is any sufficiently small constant). Certainly, Gibbs measures may not exist for an arbitrary function $\psi$ but they exist 
for a large class of continuous functions (for details, see for example [2]). It follows from our results that the set

$$
\left\{x \in X ; \liminf _{n \rightarrow \infty}-\frac{1}{n} \log \mu\left(B_{n}(x, \varepsilon)\right)<\limsup _{n \rightarrow \infty}-\frac{1}{n} \log \mu\left(B_{n}(x, \varepsilon)\right)\right\},
$$

on which the local entropy is not defined, is either empty or residual (we emphasize that the map $f$ need not be conformal).

Acknowledgment. Research supported by Portuguese National Funds through FCT - Fundação para a Ciência e a Tecnologia within the project PTDC/MAT/117106/2010 and by CAMGSD. Jinjun Li was also supported by NSFC 11301473, the NSFFP (2014J05008), the ECFP (JA13203) and the program for NCETs in Minnan Normal University (MX13002).

2. Preliminaries. Let $\sigma: \Sigma^{+} \rightarrow \Sigma^{+}$be the shift map on the space of one-sided sequences $\Sigma^{+}=\{1, \ldots, k\}^{N}$, where $k \geq 2$ is an integer. We equip $\Sigma^{+}$with the distance

$$
d\left(\omega, \omega^{\prime}\right)=2^{-n}, \quad \omega=\left(\omega_{i}\right)_{i \in N}, \quad \omega^{\prime}=\left(\omega_{i}^{\prime}\right)_{i \in N},
$$

where $n$ is the smallest integer such that $\omega_{n} \neq \omega_{n}^{\prime}$.

Given a compact and positively $\sigma$-invariant set $X \subset \Sigma^{+}$(this means that $\sigma(X) \subset X$ ) we consider the subshift $\sigma \mid X: X \rightarrow X$. For each $n \in N$, let

$$
X^{n}=\left\{\left(\omega_{1} \cdots \omega_{n}\right) ;\left(\omega_{1} \omega_{2} \cdots\right) \in X\right\} \quad \text { and } \quad X^{*}=\bigcup_{n \in N} X^{n}
$$

be, respectively, the family of all $X$-admissible strings of length $n$ and the family of all $X$ admissible finite strings. When $\omega=\left(\omega_{1} \omega_{2} \cdots\right) \in X$ and $m \in N$ or when $\omega=\left(\omega_{1} \cdots \omega_{n}\right) \in$ $X^{n}$ and $m \in N$ with $m \leq n$, we write

$$
\omega \mid m=\omega_{1} \cdots \omega_{m} .
$$

Moreover, for each $\omega \in X^{n}$, we write $|\omega|=n$ and we define the cylinder set generated by $\omega$ by

$$
[\omega]=\{\rho \in X ; \rho \mid n=\omega\} .
$$

Now we introduce the notion of weak specification. Given strings $\omega=\left(\omega_{1} \cdots \omega_{n}\right) \in X^{n}$ and $\omega^{\prime}=\left(\omega_{1}^{\prime} \cdots \omega_{m}^{\prime}\right) \in X^{m}$, let

$$
\omega \omega^{\prime}=\left(\omega_{1} \cdots \omega_{n} \omega_{1}^{\prime} \cdots \omega_{m}^{\prime}\right)
$$

be the concatenation of $\omega$ and $\omega^{\prime}$. We note that, in general, $\omega \omega^{\prime}$ need not belong to $X^{n+m}$. We say that the subshift $\sigma \mid X$ has the weak specification property if there exists $\tau \in N \cup\{0\}$ such that for any two admissible strings $\omega, \omega^{\prime} \in X^{*}$ there exists

$$
\rho=\rho\left(\omega, \omega^{\prime}\right) \in \bigcup_{k=0}^{\tau} X^{k}
$$

such that $\omega \rho \omega^{\prime} \in X^{*}$. The string $\rho\left(\omega, \omega^{\prime}\right)$ is called a bridge between $\omega$ and $\omega^{\prime}$. To simplify the notation, we write $\omega \rho \omega^{\prime}=\omega \bowtie \omega^{\prime}$ (although we emphasize that $\rho$ need not be unique). Moreover, given subsets $W, W_{1}, \ldots, W_{n}$ of $X^{*}$ and a string $\omega \in X^{*}$, we write

$$
W_{1} \bowtie \cdots \bowtie W_{n}=\left\{\omega_{1} \bowtie \omega_{2} \bowtie \cdots \bowtie \omega_{n} ; \omega_{i} \in W_{i}, 1 \leq i \leq n\right\}
$$


and

$$
\omega \bowtie W=\{\omega \bowtie \eta ; \eta \in W\},
$$

where each symbol $\bowtie$ runs over all admissible bridges. Finally, we write

$$
W^{\bowtie n}=W_{1} \bowtie \cdots \bowtie W_{n}
$$

when $W_{1}=\cdots=W_{n}=W$.

EXAMPLE 2.1. A particular class of subshifts with the weak specification property are those with the specification property, that is, those for which all bridges have the same length.

EXAMPLE 2.2. Given a $k \times k$ matrix $A=\left(a_{i j}\right)$ with entries in $\{0,1\}$, let

$$
\Sigma_{A}^{+}=\left\{\left(\omega_{1} \omega_{2} \cdots\right) \in \Sigma ; a_{\omega_{n} \omega_{n+1}}=1 \text { for } n \in N\right\} .
$$

Clearly, $\sigma\left(\Sigma_{A}^{+}\right) \subset \Sigma_{A}^{+}$. The restriction $\sigma \mid \Sigma_{A}^{+}: \Sigma_{A}^{+} \rightarrow \Sigma_{A}^{+}$is called the (one-sided) topological Markov chain or subshift of finite type with transition matrix $A$. One can easily verify that if the matrix $A$ is irreducible (that is, if for each pair $(i, j)$ there exists a power of $A$ whose $(i, j)$ th-entry is positive), then $\sigma \mid \Sigma_{A}^{+}$has the weak specification property (since there are only finitely many entries). We note that $A$ is irreducible if and only if $\sigma \mid \Sigma_{A}^{+}$is topologically transitive.

EXAMPLE 2.3. In the particular case when $A$ is transitive (that is, when some power $A^{\tau+1}$ of $A$, with $\tau \geq 0$, has only positive entries) or, equivalently, when $\sigma \mid \Sigma_{A}^{+}$is topologically mixing, the topological Markov chain $\sigma \mid \Sigma_{A}^{+}$has the specification property and all bridges (can) have length $\tau$.

EXAMPLE 2.4. A sofic shift is a continuous factor of a topological Markov chain. All sofic shifts have the specification property and are topologically mixing. We note that even though there are only countably many sofic shifts, there are uncountably many shifts with the specification property.

\section{Results for one-sided shifts.}

3.1. Main result. Let $\sigma: X \rightarrow X$ be a subshift. Given a continuous function $\varphi: X \rightarrow$ $\boldsymbol{R}$, we consider the level sets

$$
B_{\varphi}(\alpha)=\left\{\omega \in X ; \lim _{n \rightarrow \infty} S_{\varphi}(\omega, n)=\alpha\right\}
$$

where

$$
S_{\varphi}(\omega, n)=\frac{1}{n} \sum_{i=0}^{n-1} \varphi\left(\sigma^{i}(\omega)\right)
$$

We consider also the set

$$
\mathcal{L}_{\varphi}=\left\{\alpha \in \boldsymbol{R} ; B_{\varphi}(\alpha) \neq \varnothing\right\} .
$$

When $\sigma|X=\sigma| \Sigma_{A}^{+}$is a topologically mixing topological Markov chain, the set $\mathcal{L}_{\varphi}$ is a nonempty closed interval (see [9]). Moreover, one can easily verify that for each $\alpha \in A_{\varphi}(\omega)$ 
(setting $f=\sigma$ ) there exists $\omega^{\prime} \in \Sigma_{A}^{+}$such that

$$
\lim _{n \rightarrow \infty} S_{\varphi}\left(\omega^{\prime}, n\right)=\alpha
$$

(see, for example, Lemma 6.5 in [11]), that is, $\alpha \in \mathcal{L}_{\varphi}$. Therefore,

$$
\mathcal{L}_{\varphi}=\bigcup_{\omega \in \Sigma_{A}^{+}} A_{\varphi}(\omega) .
$$

The following is our main result. In fact, all other statements in the paper are its consequences.

THEOREM 3.1. Let $\sigma \mid X$ be a subshift with the weak specification property and let $\varphi: X \rightarrow \boldsymbol{R}$ be a continuous function. Given a closed interval $I \subset \mathcal{L}_{\varphi}$ that is not a singleton, if the set

$$
X_{\varphi, I}:=\left\{\omega \in X ; A_{\varphi}(\omega)=I\right\}
$$

is nonempty, then it is residual.

The proof of Theorem 3.1 is given in Section 6.

We note that if $\left(a_{n}\right)_{n}$ is a bounded sequence such that $a_{n+1}-a_{n} \rightarrow 0$ when $n \rightarrow \infty$, then its set of accumulation points $A$ is a bounded closed interval, that is,

$$
A=\left[\liminf _{n \rightarrow \infty} a_{n}, \limsup _{n \rightarrow \infty} a_{n}\right] .
$$

In particular, since

$$
\begin{aligned}
\left|S_{\varphi}(\omega, n+1)-S_{\varphi}(\omega, n)\right| & =\left|-\frac{1}{n+1} S_{\varphi}(\omega, n)+\frac{1}{n+1} \varphi\left(\sigma^{n}(\omega)\right)\right| \\
& \leq \frac{2 \max |\varphi|}{n+1} \rightarrow 0
\end{aligned}
$$

when $n \rightarrow \infty$, it is sufficient to consider closed intervals $I$ in Theorem 3.1. Moreover, by (8), we have

$$
A_{\varphi}(\omega)=\left[\liminf _{n \rightarrow \infty} S_{\varphi}(\omega, n), \limsup _{n \rightarrow \infty} S_{\varphi}(\omega, n)\right] .
$$

3.2. Applications. Now we give several applications of Theorem 3.1. The first one concerns the (irregular) set of points for which the Birkhoff averages do not converge.

THEOREM 3.2. Let $\sigma \mid X$ be a subshift with the weak specification property and let $\varphi: X \rightarrow \boldsymbol{R}$ be a continuous function. If the irregular set

$$
X_{\varphi}:=\left\{\omega \in X ; \liminf _{n \rightarrow \infty} S_{\varphi}(\omega, n)<\limsup _{n \rightarrow \infty} S_{\varphi}(\omega, n)\right\}
$$

is nonempty, then it is residual.

Proof. If the set $X_{\varphi}$ is nonempty, then there exists a closed interval $I \subset \mathcal{L}_{\varphi}$ for which $X_{\varphi, I} \neq \emptyset$ is not a singleton. Indeed, if $X_{\varphi, I}=\emptyset$ for any closed interval, then, in view of the remark after Theorem 3.1, $X_{\varphi}$ would be empty. Moreover, if for any closed interval $I$ such 
that $X_{\varphi, I} \neq \emptyset$ this last set was a singleton, then again $X_{\varphi}$ would be empty. Since $X_{\varphi, I} \subset X_{\varphi}$, the desired result follows readily from Theorem 3.1.

The former results can be extended to the case of irregular sets obtained from finitely many functions. Namely, we consider irregular sets obtained from the intersection of finitely many irregular sets as in (7).

THEOREM 3.3. Let $\sigma \mid X$ be a subshift with the weak specification property and let $\varphi_{1}, \ldots, \varphi_{k}: X \rightarrow \boldsymbol{R}$ be continuous functions. Given closed intervals $I_{i} \subset \mathcal{L}_{\varphi_{i}}$, for $i=$ $1, \ldots, k$, that are not singletons, if the set

$$
R:=X_{\varphi_{1}, I_{1}} \cap \cdots \cap X_{\varphi_{k}, I_{k}}
$$

is nonempty, then it is residual.

Proof. By Theorem 3.1, for each $i=1, \ldots, k$ there exists a $G_{\delta}$ set $E_{i} \subset X_{\varphi_{i}, I_{i}}$ that is dense in $X$. Now let

$$
F=\bigcap_{i=1}^{k} E_{i} .
$$

Clearly, the $G_{\delta}$ set $F$ is dense in $X$ and $F \subset R$. Therefore, $R$ is residual.

An argument similar to that in the proof of Theorem 3.2 yields the following result.

THEOREM 3.4. Let $\sigma \mid X$ be a subshift with the weak specification property and let $\varphi_{1}, \ldots, \varphi_{k}: X \rightarrow \boldsymbol{R}$ be continuous functions. If the irregular set

$$
S:=X_{\varphi_{1}} \cap \cdots \cap X_{\varphi_{k}}
$$

is nonempty, then it is residual.

It was observed by Barreira and Schmeling in [6] that $S$ is a proper dense set whenever it is nonempty.

4. Results for two-sided shifts. In this section we consider the shift map $\sigma: \Sigma \rightarrow \Sigma$ on the space of two-sided sequences $\Sigma=\{1, \ldots, k\}^{Z}$. We equip $\Sigma$ with the distance

$$
d\left(\omega, \omega^{\prime}\right)=2^{-\inf \left\{|n| ; \omega_{n} \neq \omega_{n}^{\prime}\right\}}, \quad \omega=\left(\omega_{i}\right)_{i \in \boldsymbol{Z}}, \quad \omega^{\prime}=\left(\omega_{i}^{\prime}\right)_{i \in \boldsymbol{Z}} .
$$

Let $\sigma: X \rightarrow X$ be a subshift, where $X \subset \Sigma$ is compact and $\sigma$-invariant. Given a continuous function $\varphi: X \rightarrow \boldsymbol{R}$, we consider the level sets $B_{\varphi}(\alpha)$ in (5) with $S_{\varphi}(\omega, n)$ as in (6). We emphasize that now each set $B_{\varphi}(\alpha)$ is composed of two-sided sequences.

The following result is a version of Theorem 3.1 for two-sided sequences.

THEOREM 4.1. Let $\sigma \mid X$ be a (two-sided) subshift with the weak specification property and let $\varphi: X \rightarrow \boldsymbol{R}$ be a continuous function. Given a closed interval $I \subset \mathcal{L}_{\varphi}$ that is not a singleton, if the set $X_{\varphi, I}$ is nonempty, then it is residual.

Proof. Let $\pi: X \rightarrow \Sigma^{+}$, be the projection defined by

$$
\pi\left(\cdots \omega_{-1} \omega_{0} \omega_{1} \cdots\right)=\left(\omega_{0} \omega_{1} \cdots\right) .
$$


By a result of Bowen in [7] (see also Section 4.2.3 in [2]), there exists a continuous function $\psi: X^{+} \rightarrow \boldsymbol{R}$, where $X^{+}=\pi(X)$, such that $\varphi$ and $\psi \circ \pi$ are cohomologous on $X$. This means that there exists a bounded function $h: X \rightarrow \boldsymbol{R}$ such that

$$
\varphi-\psi \circ \pi=h-h \circ \sigma \text { on } X \text {. }
$$

This implies that $\varphi$ and $\psi \circ \pi$ have the same irregular sets, that is,

$$
X_{\varphi, I}=X_{\psi \circ \pi, I}
$$

Now we show that

$$
X_{\psi \circ \pi, I} \supset \pi^{-1} X_{\psi, I}
$$

Let

$$
\omega=\left(\cdots \omega_{-1} \omega_{0} \omega_{1} \cdots\right) \in \pi^{-1} X_{\psi, I}
$$

with $\eta=\left(\omega_{0} \omega_{1} \cdots\right) \in X_{\psi, I}$. Then

$$
\frac{1}{n} \sum_{k=0}^{n-1}(\psi \circ \pi)\left(\sigma^{k}(\omega)\right)=\frac{1}{n} \sum_{k=0}^{n-1} \psi\left(\sigma^{k}(\eta)\right),
$$

which implies that $\omega \in X_{\psi \circ \pi, I}$. This establishes (11).

On the other hand, it follows from Theorem 3.1 that $X_{\psi, I}$ is residual, which implies that $\pi^{-1} X_{\psi, I}$ is also residual. Indeed, since $\pi$ is continuous, its preimage of a $G_{\delta}$ set is still a $G_{\delta}$ set. Moreover, it follows from the explicit form of $\pi$ in (9) that the preimage of a dense set is dense. The theorem follows now readily from (10) and (11).

The statements in Theorems 3.3 and 3.4 also hold in the context of two-sided shifts. The proofs are identical to those for one-sided shifts but now using Theorem 4.1 instead of Theorem 3.1.

5. Results for repellers. In this section we obtain corresponding results for the Birkhoff averages of a continuous function on a repeller.

Let $f: M \rightarrow M$ be a $C^{1}$ map on a smooth manifold and let $J \subset M$ be a compact $f$-invariant set (this means that $f^{-1} J=J$ ). We say that $f$ is expanding on $J$ and that $J$ is a repeller for $f$ if there exist $c>0$ and $\tau>1$ such that

$$
\left\|d_{x} f^{n} v\right\| \geq c \tau^{n}\|v\|
$$

for $x \in J, v \in T_{x} M$ and $n \in N$. We shall always assume that there exists an open neighborhood $U$ of $J$ such that $J=\bigcap_{n=0}^{\infty} f^{-n} U$. Given a continuous function $\varphi: J \rightarrow \boldsymbol{R}$, we consider the irregular set

$$
J_{\varphi}=\left\{x \in J ; \liminf _{n \rightarrow \infty} S_{\varphi}(x, n)<\limsup _{n \rightarrow \infty} S_{\varphi}(x, n)\right\},
$$

with $S_{\varphi}(x, n)$ as in (4).

The following is a version of Theorem 3.2 for the Birkhoff averages of a continuous function on a repeller. 
THEOREM 5.1. Let $J$ be a repeller for a topologically transitive $C^{1}$ map $f$ and let $\varphi: J \rightarrow \boldsymbol{R}$ be a continuous function. Then the irregular set $J_{\varphi}$ is either empty or residual.

In a manner similar to that in Section 3 we also present a more general result from which Theorem 5.1 can be deduced. Given a set $I \subset \boldsymbol{R}$, let

$$
J_{\varphi, I}=\left\{x \in X ; A_{\varphi}(x)=I\right\},
$$

where $A_{\varphi}(x)$ is the set of accumulation points of the sequence $n \mapsto S_{\varphi}(x, n)$. Moreover, let

$$
\mathcal{R}_{\varphi}=\left\{\alpha \in \boldsymbol{R} ; J_{\varphi}(\alpha) \neq \varnothing\right\},
$$

where

$$
J_{\varphi}(\alpha)=\left\{x \in J ; \lim _{n \rightarrow \infty} S_{\varphi}(x, n)=\alpha\right\} .
$$

THEOREM 5.2. Let $J$ be a repeller for a topologically transitive $C^{1}$ map and let $\varphi: J \rightarrow \boldsymbol{R}$ be a continuous function. Given a closed interval $I \subset \mathcal{R}_{\varphi}$ that is not a singleton, if the set $J_{\varphi, I}$ is nonempty, then it is residual on $J$.

PROOF. Recall that a collection of closed sets $R_{1}, \ldots, R_{k} \subset J$ is called a Markov partition of $J$ (with respect to $f$ ) if:

(1) $J=\bigcup_{i=1}^{k} R_{i}$ and $R_{i}=\overline{\operatorname{int} R_{i}}$ for each $i$;

(2) int $R_{i} \cap$ int $R_{j}=\emptyset$ whenever $i \neq j$;

(3) if int $f\left(R_{i}\right) \cap$ int $R_{j} \neq \emptyset$, then $f\left(R_{i}\right) \supset R_{j}$.

We note that the interiors are computed with respect to the induced topology on $J$. Any repeller $J$ for a $C^{1}$ map $f$ has Markov partitions of arbitrary small diameter (see for example [3]). Let $A=\left(a_{i j}\right)$ be a $k \times k$ matrix with entries $a_{i j}=1$ if int $f\left(R_{i}\right) \cap$ int $R_{j} \neq \emptyset$ and $a_{i j}=0$ otherwise. Writing $X=\Sigma_{A}^{+}$, we obtain a coding map $\pi: X \rightarrow J$ for the repeller $J$ letting

$$
\pi(\omega)=\bigcap_{n \in N} f^{-n+1} R_{\omega_{n}}, \quad \omega=\left(\omega_{1} \omega_{2} \cdots\right) .
$$

One can easily verify that $\pi$ is continuous, onto and that $\pi \circ \sigma=f \circ \pi$ on $X$. The last identity implies that $\mathcal{R}_{\varphi}=\mathcal{L}_{\varphi \circ \pi}$.

Now let

$$
B=\bigcup_{n \geq 0} f^{-n} \bigcup_{i=1}^{k} \partial R_{i},
$$

where $\partial R_{i}$ is the boundary of $R_{i}$. This is the set of points in $J$ for which the coding is not unique. Since $f(C) \subset C$, where $C=\bigcup_{i=1}^{k} \partial R_{i}$, the sequence $f^{-n} C$ is increasing and hence the set $B$ is invariant, that is, $(f \mid J)^{-1} B=B$. We define

$$
S=X \backslash \pi^{-1} B \text { and } \quad J^{*}=J \backslash B .
$$

Clearly, the map $\pi: S \rightarrow J^{*}$ is bijective. Moreover, $B$ is an $F_{\sigma}$ set and since $\pi$ is continuous, $S$ is a $G_{\delta}$ set. In addition, it follows from the $f$-invariance of $B$ that $(f \mid J)^{-1} J^{*}=J^{*}$ and hence $(\sigma \mid X)^{-1} S=S$. Since any nonempty invariant set (not forward invariant set) of a onesided topologically transitive topological Markov chain $\sigma \mid X$ is dense, $S$ is a dense $G_{\delta}$ set. 
We note that $\psi=\varphi \circ \pi$ is a continuous function on $X$. Let $I \subset \mathcal{R}_{\varphi}=\mathcal{L}_{\psi}$ be a closed interval. It follows from Theorem 3.1 that there exists a dense $G_{\delta}$ set $E \subset X_{\psi, I}$. To complete the proof, it suffices to show that the set $F=\pi(E \cap S) \subset J^{*}$ satisfies the following properties:

(1) $F \subset J_{\varphi, I}$

(2) $F$ is dense in $J$;

(3) $F$ is a $G_{\delta}$ set.

It follows from the identity $\pi \circ \sigma=f \circ \pi$ that

$$
F \subset \pi(E) \subset \pi\left(X_{\psi, I}\right)=J_{\varphi, I} .
$$

Moreover, $E \cap S$ is a dense $G_{\delta}$ set since both $E$ and $S$ are dense $G_{\delta}$ sets. In particular,

$$
J=\pi(X)=\pi(\overline{E \cap S}) \subset \overline{\pi(E \cap S)}=\bar{F}
$$

and $F$ is dense in $J$. For the last property, we observe that

$$
\begin{aligned}
J \backslash F & =\left(B \cup J^{*}\right) \backslash F=B \cup\left(J^{*} \backslash F\right) \quad(\text { since } B \cap F=\emptyset) \\
& =B \cup(\pi(S) \backslash \pi(E \cap S)) \\
& =B \cup \pi(S \backslash(E \cap S)) \quad(\text { since } \pi \text { is bijective on } S) \\
& =\pi(X \backslash S) \cup \pi(S \backslash(E \cap S)) \\
& =\pi((X \backslash S) \cup(S \backslash(E \cap S))) \\
& =\pi(X \backslash(E \cap S)) .
\end{aligned}
$$

Finally, $X \backslash(E \cap S)$ is an $F_{\sigma}$ set (since $E \cap S$ is a $G_{\delta}$ set) and writing $X \backslash(E \cap S)=\bigcup_{i} F_{i}$ as a countable union of closed sets $F_{i} \subset X$, we obtain

$$
J \backslash F=\pi(X \backslash(E \cap S))=\bigcup_{i} \pi\left(F_{i}\right),
$$

where $\pi\left(F_{i}\right)$ is a closed set (since $\pi$ is continuous and $X$ is compact). This shows that $F$ is a $G_{\delta}$ set and the proof of the theorem is complete.

6. Proof of Theorem 3.1. In order to prove Theorem 3.1 we construct a dense $G_{\delta}$ set $E \subset X$ such that $E \subset X_{\varphi, I}$.

For each $\alpha \in \boldsymbol{R}, n \in \boldsymbol{N}$ and $\varepsilon>0$, let

$$
F(\alpha, n, \varepsilon)=\left\{\omega \mid n ; \omega \in X \text { and }\left|S_{\varphi}(\omega, n)-\alpha\right|<\varepsilon\right\},
$$

with $S_{\varphi}(\omega, n)$ as in (6). Given $\varepsilon>0$, we have $F(\alpha, n, \varepsilon) \neq \emptyset$ for each $\alpha \in \mathcal{L}_{\varphi}$ and any sufficiently large $n$ (depending on $\alpha$ and $\varepsilon$ ).

Now choose numbers $\alpha_{k, 1}, \ldots, \alpha_{k, q_{k}} \in I$ for each $k \in N$ such that

$$
I \subset \bigcup_{i=1}^{q_{k}} B\left(\alpha_{k, i}, 1 / k\right)
$$

and

$$
\left|\alpha_{k, i+1}-\alpha_{k, i}\right|<\frac{1}{k} \text { for } i=0, \ldots, q_{k}-1, \quad\left|\alpha_{k, q_{k}}-\alpha_{k+1,1}\right|<\frac{1}{k}
$$


Moreover, let $\varepsilon_{1}>\varepsilon_{2}>\cdots$ be a sequence of positive numbers decreasing to zero and let

$$
n_{1,1}<n_{1,2}<\cdots<n_{1, q_{1}}<n_{2,1}<n_{2,2}<\cdots<n_{2, q_{2}}<\cdots
$$

be positive integers such that

$$
F\left(\alpha_{k, i}, n_{k, i}, \varepsilon_{k}\right) \neq \emptyset \quad \text { for } \quad k \in N, 1 \leq i \leq q_{k} .
$$

Let $\Omega_{0}=X^{*}$. For each $\omega \in \Omega_{0}$, we take integers $\left\{N_{k, i}(\omega)\right\}_{k \in N, i=1, \ldots, q_{k}}$ such that the following conditions are satisfied:

(i) $N_{1, i}(\omega) \geq 2^{n_{1, i+1}+\tau}$ for $2 \leq i \leq q_{1}-1$,

$$
\begin{aligned}
& N_{k, i}(\omega) \geq 2^{n_{k, i+1}+\tau} \text { for } k \geq 2 \text { and } 1 \leq i \leq q_{k}-1, \\
& N_{k, q_{k}}(\omega) \geq 2^{n_{k+1,1}+\tau} \text { for } k \geq 1 ;
\end{aligned}
$$

(ii) $N_{k, i+1}(\omega) \geq 2^{|\omega|+\tau+N_{1,1}(\omega)\left(n_{1,1}+\tau\right)+N_{1,2}(\omega)\left(n_{1,2}+\tau\right)+\cdots+N_{k, i}(\omega)\left(n_{k, i}+\tau\right)}$,

$$
N_{k+1,1}(\omega) \geq 2^{|\omega|+\tau+N_{1,1}(\omega)\left(n_{1,1}+\tau\right)+N_{1,2}(\omega)\left(n_{1,2}+\tau\right)+\cdots+N_{k, q_{k}}(\omega)\left(n_{k, q_{k}}+\tau\right)}
$$

for $k \in N$ and $i=1, \ldots, q_{k}-1$.

We recall that $\tau$ is the integer in the notion of weak specification (see Section 2). Now we define recursively sets $\Omega_{k, i} \subset X^{*}$ for $k \in N$ and $i=1, \ldots, q_{k}$ by

$$
\begin{aligned}
\Omega_{1,1} & =\bigcup_{\omega \in \Omega_{0}} \omega \bowtie F\left(\alpha_{1,1}, n_{1,1}, \varepsilon_{1}\right)^{\bowtie N_{1,1}(\omega)}, \\
\Omega_{1,2} & =\bigcup_{\eta \in \Omega_{1,1}} \eta \bowtie F\left(\alpha_{1,2}, n_{1,2}, \varepsilon_{1}\right)^{\bowtie N_{1,2}(\omega)}, \\
& \cdots \\
\Omega_{1, q_{1}} & =\bigcup_{\eta \in \Omega_{1, q_{1}-1}} \eta \bowtie F\left(\alpha_{1, q_{1}}, n_{1, q_{1}}, \varepsilon_{1}\right)^{\bowtie N_{1, q_{1}}(\omega),} \\
\Omega_{2,1} & =\bigcup_{\eta \in \Omega_{1, q_{1}}} \eta \bowtie F\left(\alpha_{2,1}, n_{2,1}, \varepsilon_{2}\right)^{\bowtie N_{2,1}(\omega)},
\end{aligned}
$$

and so on (taking some bridge at each concatenation). Finally, let

$$
E_{k, i}=\bigcup_{\omega \in \Omega_{k, i}}[\omega]
$$

and

$$
E=\bigcap_{k=1}^{\infty} \bigcap_{i=1}^{q_{k}} E_{k, i} .
$$

Clearly, $E$ is a $G_{\delta}$ set since each cylinder set $[\omega]$ is open.

Now we show that $E$ has the desired properties.

LEMMA 6.1. E is dense in $X$.

Proof. It suffices to show that $E \cap B(\omega, r) \neq \emptyset$ for every $\omega \in X$ and $r>0$, where $B(\omega, r)$ is the ball of radius $r$ centered at $\omega$. We first observe that $X_{\varphi}$ is dense on $X$. Indeed, since $\mathcal{L}_{\varphi}$ is not a singleton, the set $X_{\varphi}$ is nonempty. Moreover, $\sigma^{-n} X_{\varphi} \subset X_{\varphi}$ for $n \in N$ and $\sigma^{-n} X_{\varphi}$ intersects all cylinder sets of length $n-\tau$ for each $n>\tau$ (with $\tau$ as in the definition 
of weak specification). Therefore, given $\omega \in X$ and $r>0$, there exist $\omega^{\prime} \in X_{\varphi}$ and $n \in N$ such that $\left[\omega^{\prime} \mid n\right] \subset B(\omega, r)$. Write $\eta=\omega^{\prime} \mid n$. Clearly, $\eta \in \Omega_{0}$. We take

$$
\begin{aligned}
\eta_{1,1} & \in \eta \bowtie F\left(\alpha_{1,1}, n_{1,1}, \varepsilon_{1}\right)^{\bowtie N_{1,1}(\eta)}, \\
\eta_{1,2} & \in \eta_{1,1} \bowtie F\left(\alpha_{1,2}, n_{1,2}, \varepsilon_{1}\right)^{\bowtie N_{1,2}(\eta)}, \\
& \ldots \\
\eta_{1, q_{1}} & \in \eta_{1, q_{1}-1} \bowtie F\left(\alpha_{1, q_{1}}, n_{1, q_{1}}, \varepsilon_{1}\right)^{\bowtie N_{1, q_{1}}(\eta),} \\
\eta_{2,1} & \in \eta_{1, q_{1}} \bowtie F\left(\alpha_{2,1}, n_{2,1}, \varepsilon_{2}\right)^{\bowtie N_{2,1}(\eta)},
\end{aligned}
$$

and so on (taking some bridge at each concatenation).

It is easy to check that $\left(\left[\eta_{k, i}\right]\right)_{k \in N, i=1, \ldots, q_{k}}$ is a decreasing sequence of nonempty compact subsets of $X$ and thus,

$$
S:=\bigcap_{k, i}\left[\eta_{k, i}\right] \cap[\eta] \neq \varnothing .
$$

Let $\rho \in S$. We claim that $\rho \in E \cap B(\omega, r)$. Indeed, it follows from the inclusion $S \subset \bigcap_{k, i} E_{k, i}$ that $\rho \in E$. On the other hand,

$$
S \subset[\eta] \subset B(\omega, r)
$$

and hence $\rho \in B(\omega, r)$.

Alternatively, one can observe that by construction each set $E_{k, i}$ is dense and so it follows from Baire's theorem that $E$ is dense (since it is a countable intersection of open dense sets in a complete metric space).

LEMMA 6.2. $E \subset X_{\varphi, I}$.

Proof. In order to prove that $E \subset X_{\varphi, I}$, we must show that $A_{\varphi}(\omega)=I$ for $\omega \in E$. We recall that for each $\omega \in E$, there exists $\omega^{0} \in \Omega_{0}$ such that

$$
\omega \in \omega^{0} \bowtie F\left(\alpha_{1,1}, n_{1,1}, \varepsilon_{1}\right)^{\bowtie N_{1,1}\left(\omega^{0}\right)} \bowtie \cdots .
$$

We first show that

$$
I \subset A_{\varphi}(\omega)
$$

Given

$$
\alpha \in I \subset \bigcup_{i=1}^{q_{k}} B\left(\alpha_{k, i}, 1 / k\right),
$$

take an integer $i_{k} \in\left\{1, \ldots, q_{k}\right\}$ such that $\alpha \in B\left(\alpha_{k, i_{k}}, 1 / k\right)$. In order to avoid a tedious notation we assume that $i_{k} \notin\left\{1, q_{k}\right\}$ although the argument is identical when $i_{k} \in\left\{1, q_{k}\right\}$. Let 
(16)

$$
s_{k, i_{k}}=\left|\omega^{0}\right|+\lambda_{0}+\sum_{j=1}^{q_{1}} \sum_{l=1}^{N_{1, j}}\left(n_{1, j}+\lambda_{1, j, l}\right)+\cdots+\sum_{j=1}^{i_{k}} \sum_{l=1}^{N_{k, j}}\left(n_{k, j}+\lambda_{k, j, l}\right),
$$

where $\lambda_{0}$ is the length of the first bridge, $N_{k, j}=N_{k, j}\left(\omega^{0}\right)$ and

$$
\lambda_{i, j, l}= \begin{cases}\tau_{i, j} & \text { if } l \neq N_{i, j} \\ \tau_{i, j}^{\prime} & \text { if } l=N_{i, j}\end{cases}
$$

is the length of each successive bridge between strings of $\omega^{0}$. It follows from weak specification that the numbers $\lambda_{0}$ and $\lambda_{i, j, l}$ are bounded by $\tau$.

We will prove that

$$
\left|S_{\varphi}\left(\omega, s_{k, i_{k}}\right)-\alpha_{k, i_{k}}\right| \rightarrow 0 \quad \text { when } k \rightarrow \infty
$$

We first show that (17) implies (15). Indeed, it follows from (17) that

$$
\begin{aligned}
\left|S_{\varphi}\left(\omega, s_{k, i_{k}}\right)-\alpha\right| & \leq\left|S_{\varphi}\left(\omega, s_{k, i_{k}}\right)-\alpha_{k, i_{k}}\right|+\left|\alpha_{k, i_{k}}-\alpha\right| \\
& <\left|S_{\varphi}\left(\omega, s_{k, i_{k}}\right)-\alpha_{k, i_{k}}\right|+\frac{1}{k} \rightarrow 0
\end{aligned}
$$

when $k \rightarrow \infty$. Therefore, $\alpha \in A_{\varphi}(\omega)$ and (15) holds.

In order to prove (17), write

$$
s_{k, i_{k}}=\widetilde{s}_{k, i_{k}}+t_{k, i_{k}}
$$

where

$$
t_{k, i_{k}}=\sum_{l=1}^{N_{k, i_{k}}}\left(n_{k, i_{k}}+\lambda_{k, i_{k}, l}\right)
$$

Since

$$
\alpha_{k, i_{k}} \in[-\|\varphi\|,\|\varphi\|], \quad \text { where } \quad\|\varphi\|=\max _{\omega \in X}|\varphi(\omega)|,
$$


we obtain

$$
\begin{aligned}
\left|\sum_{i=0}^{s_{k, i_{k}}-1} \varphi\left(\sigma^{i}(\omega)\right)-s_{k, i_{k}} \alpha_{k, i_{k}}\right| & \\
\leq & \left|\sum_{i=0}^{\widetilde{s}_{k, i_{k}}-1} \varphi\left(\sigma^{i}(\omega)\right)-\widetilde{s}_{k, i_{k}} \alpha_{k, i_{k}}\right|+\left|\sum_{i=\widetilde{s}_{k, i_{k}}}^{s_{k, i_{k}}-1} \varphi\left(\sigma^{i}(\omega)\right)-t_{k, i_{k}} \alpha_{k, i_{k}}\right| \\
\leq & 2 \widetilde{s}_{k, i_{k}}\|\varphi\|+\left|\sum_{i=0}^{t_{k, i_{k}}-1} \varphi\left(\sigma^{i}\left(\sigma^{\widetilde{s}_{k, i_{k}}}(\omega)\right)\right)-t_{k, i_{k}} \alpha_{k, i_{k}}\right| \\
\leq & 2 \widetilde{s}_{k, i_{k}}\|\varphi\|+\sum_{l=1}^{N_{k, i_{k}}}\left|\sum_{j=0}^{n_{k, i_{k}}-1} \varphi\left(\sigma^{j}\left(\sigma^{\widetilde{s}_{k, i_{k}}+(l-1)\left(n_{k, i_{k}}+\lambda_{k, i_{k}, l}\right)}(\omega)\right)\right)-n_{k, i_{k}} \alpha_{k, i_{k}}\right| \\
& +\sum_{l=1}^{N_{k, i_{k}}}\left|\sum_{j=n_{k, i_{k}}}^{n_{k, i_{k}}+\lambda_{i, i_{k}, l}-1} \varphi\left(\sigma^{j}\left(\sigma^{\widetilde{s}_{k, i_{k}}+(l-1)\left(n_{k, i_{k}}+\lambda_{k, i_{k}, l}\right)}(\omega)\right)\right)\right|+\left|N_{k, i_{k}} \tau \alpha_{k, i_{k}}\right| \\
\leq & 2 \widetilde{s}_{k, i_{k}}\|\varphi\|+\sum_{l=1}^{N_{k, i_{k}}}\left|\sum_{j=0}^{n_{k, i_{k}}-1} \varphi\left(\sigma^{j}\left(\sigma^{\widetilde{s}_{k, i_{k}}+(l-1)\left(n_{k, i_{k}}+\lambda_{k, i_{k}, l}\right)}(\omega)\right)\right)-n_{k, i_{k}} \alpha_{k, i_{k}}\right| \\
& +2 \tau N_{k, i_{k}}\|\varphi\|
\end{aligned}
$$

In order to estimate the second term in the right-hand side of the former inequality, we introduce the numbers

$$
v_{n}(\varphi)=\sup \left\{\left|\varphi(\omega)-\varphi\left(\omega^{\prime}\right)\right| ; \omega, \omega^{\prime} \in X, \omega\left|n=\omega^{\prime}\right| n\right\}
$$

and

$$
V_{n}(\varphi)=\sum_{j=1}^{n} v_{j}(\varphi)
$$

By (14) and the definition of the set $F\left(\alpha_{k, i_{k}}, n_{k, i_{k}}, \varepsilon_{k}\right)$, one can choose $\bar{\omega}^{1}, \ldots, \bar{\omega}^{N_{k, i_{k}}} \in X$ such that

$$
\widetilde{\sigma}^{\widetilde{s}_{k, i_{k}}+(l-1)\left(n_{k, i_{k}}+\lambda_{k, i_{k}, l}\right)}(\omega)\left|n_{k, i_{k}}=\bar{\omega}^{l}\right| n_{k, i_{k}}
$$

and

$$
\left|S_{\varphi}\left(\bar{\omega}^{l}, n_{k, i_{k}}\right)-\alpha_{k, i_{k}}\right|<\varepsilon_{k}
$$


for $l=1, \ldots, N_{k, i_{k}}$. It follows from (20) and (21) that

$$
\begin{aligned}
& \left|\sum_{j=0}^{n_{k, i_{k}}-1} \varphi\left(\sigma^{j}\left(\sigma^{\widetilde{s}_{k, i_{k}}+(l-1)\left(n_{k, i_{k}}+\lambda_{k, i_{k}, l}\right)}(\omega)\right)\right)-n_{k, i_{k}} \alpha_{k, i_{k}}\right| \\
& \leq\left|\sum_{j=0}^{n_{k, i_{k}}-1} \varphi\left(\sigma^{j}\left(\sigma^{\widetilde{s}_{k, i_{k}}+(l-1)\left(n_{k, i_{k}}+\lambda_{k, i_{k}, l}\right)}(\omega)\right)\right)-\sum_{j=0}^{n_{k, i_{k}}-1} \varphi\left(\sigma^{j}\left(\bar{\omega}^{l}\right)\right)\right| \\
& \quad+\left|\sum_{j=0}^{n_{k, i_{k}}-1} \varphi\left(\sigma^{j}\left(\bar{\omega}^{l}\right)\right)-n_{k, i_{k}} \alpha_{k, i_{k}}\right| \\
& \leq V_{n_{k, i_{k}}}(\varphi)+n_{k, i_{k}} \varepsilon_{k}
\end{aligned}
$$

for $l=1, \ldots, N_{k, i_{k}}$. Together with (19) this implies that

$$
\begin{aligned}
& \left|\sum_{i=0}^{s_{k, i_{k}}-1} \varphi\left(\sigma^{i}(\omega)\right)-s_{k, i_{k}} \alpha_{k, i_{k}}\right| \\
& \quad \leq 2 \widetilde{s}_{k, i_{k}}\|\varphi\|+N_{k, i_{k}}\left(V_{n_{k, i_{k}}}(\varphi)+1\right)+2 \tau N_{k, i_{k}}\|\varphi\| \\
& \quad=2 \widetilde{s}_{k, i_{k}}\|\varphi\|+N_{k, i_{k}} V_{n_{k, i_{k}}}(\varphi)+N_{k, i_{k}}\left(n_{k, i_{k}} \varepsilon_{k}+2 \tau\|\varphi\|\right) .
\end{aligned}
$$

Now we observe that it follows from condition (ii) that $\widetilde{s}_{k, i_{k}} / s_{k, i_{k}}$ tends to zero when $k \rightarrow \infty$. Indeed, using (16), (18) and condition (ii), we have

$$
\frac{s_{k, i_{k}}}{\widetilde{s}_{k, i_{k}}}-1=\frac{t_{k, i_{k}}}{\widetilde{s}_{k, i_{k}}} \geq \frac{N_{k, i_{k}}}{\widetilde{s}_{k, i_{k}}} n_{k, i_{k}} \geq \frac{2^{\widetilde{s}_{k, i_{k}}}}{\widetilde{s}_{k, i_{k}}} n_{k, i_{k}}
$$

and thus,

$$
\frac{s_{k, i_{k}}}{\widetilde{s}_{k, i_{k}}} \rightarrow+\infty \text { when } k \rightarrow \infty .
$$

Moreover, it follows readily from the uniform continuity of $\varphi$ on the compact set $X$ that $v_{n}(\varphi) \rightarrow 0$ when $n \rightarrow \infty$. Hence, $V_{n}(\varphi) / n \rightarrow 0$ when $n \rightarrow \infty$ and

$$
\frac{N_{k, i_{k}} V_{n_{k, i_{k}}}(\varphi)}{s_{k, i_{k}}} \leq \frac{V_{n_{k, i_{k}}}(\varphi)}{n_{k, i_{k}}} \rightarrow 0 \text { when } k \rightarrow \infty .
$$

Finally, by the definition of $s_{k, i_{k}}$ (see (18)), we have $s_{k, i_{k}}>t_{k, i_{k}}$ and

$$
\frac{N_{k, i_{k}} n_{k, i_{k}} \varepsilon_{k}}{s_{k, i_{k}}}<\frac{N_{k, i_{k}} n_{k, i_{k}} \varepsilon_{k}}{t_{k, i_{k}}} \leq \varepsilon_{k} .
$$

Therefore,

$$
\left|S_{\varphi}\left(\omega, s_{k, i_{k}}\right)-\alpha_{k, i_{k}}\right|<\frac{2 \widetilde{s}_{k, i_{k}}\|\varphi\|}{s_{k, i_{k}}}+\frac{V_{n_{k, i_{k}}}(\varphi)}{n_{k, i_{k}}}+\frac{n_{k, i_{k}} \varepsilon_{k}+2 \tau\|\varphi\|}{n_{k, i_{k}}} \rightarrow 0
$$

when $k \rightarrow \infty$, which completes the proof of (17).

Now we show that

$$
A_{\varphi}(\omega) \subset I
$$


For each positive integer $n>\left|\omega^{0}\right|+\tau$ there exist $k \in N, i_{k} \in\left\{1,2, \ldots, q_{k}\right\}$ and $1 \leq p \leq$ $N_{k, i_{k}+1}$ such that

$$
s_{k, i_{k}}+\rho_{p}<n \leq s_{k, i_{k}}+\rho_{p+1},
$$

where

$$
\rho_{s}=\sum_{l=1}^{s-1}\left(n_{k, i_{k}+1}+\lambda_{k, i_{k}+1, l}\right)
$$

for each $s \in N$. Notice that $k \rightarrow \infty$ when $n \rightarrow \infty$. We claim that

$$
\left|S_{\varphi}(\omega, n)-\alpha_{k, i_{k}}\right| \rightarrow 0 \quad \text { when } n \rightarrow \infty
$$

For simplicity of the notation, in a similar manner to that in the former inclusion we assume that $i_{k} \neq q_{k}$. If (24) holds, then it follows from (12) that

$$
\operatorname{dist}\left(S_{\varphi}(\omega, n), I\right) \leq\left|S_{\varphi}(\omega, n)-\alpha_{k, i_{k}}\right|+\operatorname{dist}\left(\alpha_{k, i_{k}}, I\right) \rightarrow 0
$$

when $k \rightarrow \infty$. Since $I$ is closed, we conclude that (22) holds.

Now we establish property (24). We have

$$
\begin{aligned}
\left|\sum_{i=0}^{n-1} \varphi\left(\sigma^{i}(\omega)\right)-n \alpha_{k, i_{k}}\right| \leq & \left|\sum_{i=0}^{s_{k, i_{k}}-1} \varphi\left(\sigma^{i}(\omega)\right)-s_{k, i_{k}} \alpha_{k, i_{k}}\right| \\
& +\left|\sum_{i=s_{k, i_{k}}}^{s_{k, i_{k}}+\rho_{p}-1} \varphi\left(\sigma^{i}(\omega)\right)-\rho_{p} \alpha_{k, i_{k}}\right| \\
& +\left|\sum_{i=s_{k, i_{k}}+\rho_{p}}^{n-1} \varphi\left(\sigma^{i}(\omega)\right)-\left(n-s_{k, i_{k}}-\rho_{p}\right) \alpha_{k, i_{k}}\right| .
\end{aligned}
$$

We shall estimate each of these terms. In a similar manner to that in (20) and (21), one can choose $\bar{\omega}^{1}, \ldots, \bar{\omega}^{p-1} \in X$ such that

$$
\sigma^{s_{k, i_{k}}+(l-1)\left(n_{k, i_{k}+1}+\lambda_{k, i_{k}+1, l}\right)}(\omega)\left|n_{k, i_{k}+1}=\bar{\omega}^{l}\right| n_{k, i_{k}+1}
$$

and

$$
\left|S_{\varphi}\left(\bar{\omega}^{l}, n_{k, i_{k}+1}\right)-\alpha_{k, i_{k}+1}\right|<\varepsilon_{k}
$$


for $l=1, \ldots, p-1$. It follows from (13), (25) and (26) that

$$
\begin{aligned}
& \left|\sum_{j=0}^{n_{k, i_{k}+1}-1} \varphi\left(\sigma^{j}\left(\sigma^{s_{k, i_{k}}+(l-1)\left(n_{k, i_{k}+1}+\lambda_{k, i_{k}+1, l}\right)}(\omega)\right)\right)-n_{k, i_{k}+1} \alpha_{k, i_{k}}\right| \\
& \leq\left|\sum_{j=0}^{n_{k, i_{k}+1}-1} \varphi\left(\sigma^{j}\left(\sigma^{s_{k, i_{k}}+(l-1)\left(n_{k, i_{k}+1}+\lambda_{k, i_{k}+1, l}\right)}(\omega)\right)\right)-n_{k, i_{k}+1} \alpha_{k, i_{k}+1}\right| \\
& \quad+\left|n_{k, i_{k}+1} \alpha_{k, i_{k}+1}-n_{k, i_{k}+1} \alpha_{k, i_{k}}\right| \\
& \leq\left|\sum_{j=0}^{n_{k, i_{k}+1}-1} \varphi\left(\sigma^{j}\left(\sigma^{s_{k, i_{k}}+(l-1)\left(n_{k, i_{k}+1}+\lambda_{k, i_{k}+1, l}\right)}(\omega)\right)\right)-\sum_{j=0}^{n_{k, i_{k}+1}-1} \varphi\left(\sigma^{j}\left(\bar{\omega}^{l}\right)\right)\right| \\
& \quad+\left|\sum_{j=0}^{n_{k, i_{k}+1}-1} \varphi\left(\sigma^{j}\left(\bar{\omega}^{l}\right)\right)-n_{k, i_{k}+1} \alpha_{k, i_{k}+1}\right|+\frac{n_{k, i_{k}+1}}{k} \\
& \leq V_{n_{k, i_{k}+1}}(\varphi)+n_{k, i_{k}+1} \varepsilon_{k}+\frac{n_{k, i_{k}+1}}{k}
\end{aligned}
$$

for $l=1, \ldots, p-1$. Therefore,

$$
\begin{aligned}
& \left|\sum_{i=s_{k, i_{k}}}^{s_{k, i_{k}}+\rho_{p}-1} \varphi\left(\sigma^{i}(\omega)\right)-\rho_{p} \alpha_{k, i_{k}}\right| \\
& \quad \leq\left|\sum_{l=1}^{p-1}\left(\sum_{j=0}^{n_{k, i_{k}+1}-1} \varphi\left(\sigma^{j}\left(\sigma^{s_{k, i_{k}}+(l-1)\left(n_{k, i_{k}+1}+\lambda_{k, i_{k}+1, l}\right)}(\omega)\right)\right)-n_{k, i_{k}+1} \alpha_{k, i_{k}}\right)\right| \\
& \quad+2(p-1) \tau\|\varphi\| \\
& \quad \leq p\left(V_{n_{k, i_{k}+1}}(\varphi)+n_{k, i_{k}+1} \varepsilon_{k}+\frac{n_{k, i_{k}+1}}{k}\right)+2 p \tau\|\varphi\| .
\end{aligned}
$$

Moreover, by (23), we have

$$
\begin{aligned}
\left|\sum_{i=s_{k, i_{k}}+\rho_{p}}^{n-1} \varphi\left(\sigma^{i}(\omega)\right)-\left(n-s_{k, i_{k}}-\rho_{p}\right) \alpha_{k, i_{k}}\right| & \leq 2\left(n-s_{k, i_{k}}-\rho_{p}\right)\|\varphi\| \\
& \leq 2\left(n_{k, i_{k}+1}+\tau\right)\|\varphi\| .
\end{aligned}
$$

Collecting the estimates (27) and (28), we obtain

$$
\begin{aligned}
\left|S_{\varphi}(\omega, n)-\alpha_{k, i_{k}}\right| \leq & \left|S_{\varphi}\left(\omega, s_{k, i_{k}}\right)-\alpha_{k, i_{k}}\right| \frac{s_{k, i_{k}}}{n}+\frac{2\left(n_{k, i_{k}+1}+\tau\right)\|\varphi\|}{n} \\
& +\frac{p V_{n_{k, i_{k}+1}}(\varphi)}{n}+\frac{p n_{k, i_{k}+1}}{k n}+\frac{p\left(n_{k, i_{k}+1} \varepsilon_{k}+2 \tau\|\varphi\|\right)}{n} .
\end{aligned}
$$


In a manner similar to that in the proof of (17), one can show that the first term in (29) tends to zero when $n \rightarrow \infty$ (notice that $s_{k, i_{k}} \leq n$ ). Moreover, using (23) and condition (i), we obtain

$$
\frac{2\left(n_{k, i_{k}+1}+\tau\right)\|\varphi\|}{n} \leq \frac{2\left(n_{k, i_{k}+1}+\tau\right)\|\varphi\|}{s_{k, i_{k}}} \leq \frac{2\left(n_{k, i_{k}+1}+\tau\right)\|\varphi\|}{N_{k, i_{k}}} \rightarrow 0
$$

when $n \rightarrow \infty$. On the other hand, it follows from (23) that

$$
\begin{gathered}
\frac{p n_{k, i_{k}+1}}{k n} \leq \frac{1}{k} \rightarrow 0 \quad \text { when } n \rightarrow \infty, \\
\frac{p V_{n_{k, i_{k}+1}}(\varphi)}{n} \leq \frac{V_{n_{k, i_{k}+1}}(\varphi)}{n_{k, i_{k}+1}} \rightarrow 0 \text { when } n \rightarrow \infty,
\end{gathered}
$$

and

$$
\frac{p\left(n_{k, i_{k}+1} \varepsilon_{k}+2 \tau\|\varphi\|\right)}{n} \leq \frac{n_{k, i_{k}+1} \varepsilon_{k}+2 \tau\|\varphi\|}{n_{k, i_{k}+1}} \rightarrow 0 \quad \text { when } n \rightarrow \infty
$$

(since $k \rightarrow \infty$ when $n \rightarrow \infty$ ).

Hence, property (24) follows readily from (29), (30), (32), (31) and (33). This completes the proof of the lemma.

Theorem 3.1 follows immediately from Lemmas 6.1 and 6.2.

\section{REFERENCES}

[ 1 ] S. Albeverio, M. Pratsiovytyi and G. Torbin, Topological and fractal properties of subsets of real numbers which are not normal, Bull. Sci. Math. 129 (2005), 615-630.

[ 2 ] L. BARReIRA, Dimension and Recurrence in Hyperbolic Dynamics, Progress in Mathematics 272, Birkhäuser, 2008.

[ 3 ] L. BARReIRA, Ergodic Theory, Hyperbolic Dynamics and Dimension Theory, Universitext, Springer, 2012.

[ 4 ] L. BArReira AND B. SAussol, Multifractal analysis of hyperbolic flows, Comm. Math. Phys. 214 (2000), 339-371.

[ 5 ] L. BARReira, B. SAUSSOl AND J. Schmeling, Distribution of frequencies of digits via multifractal analysis, J. Number Theory 97 (2002), 410-438.

[6] L. BARREIRA AND J. SCHMELING, Sets of "non-typical" points have full topological entropy and full Hausdorff dimension, Israel J. Math. 116 (2000), 29-70.

[ 7 ] R. Bowen, Equilibrium States and the Ergodic Theory of Anosov Diffeomorphisms, Lecture Notes in Math., Vol. 470, Springer-Verlag, 1975.

[ 8 ] E. Chen, T. KÜPPer And L. Shu, Topological entropy for divergence points, Ergodic Theory. Dynam. Systems 25 (2005), 1173-1208.

[9] A.-H. FAn AND D.-J. FEnG, On the distribution of long-term time averages on symbolic space, J. Stat. Phys. 99 (2000), 813-856.

[10] A.-H. FAn, D.-J. FEng AND J. Wu, Recurrence, dimension and entropy, J. London Math. Soc. (2) 64 (2001), 229-244.

[11] D.-J. FenG, K.-S. LAU AND J. WU, Ergodic limits on the conformal repellers, Adv. Math. 169 (2002), 58-91.

[12] J. Li AND M. WU, Divergence points in systems satisfying the specification property, Discrete Contin. Dyn. Syst. 33 (2013), 905-920. 
[13] YA. Pesin AND B. Pitskel, Topological pressure and the variational principle for noncompact sets, Funktsional. Anal. i Prilozhen 18 (1984), 307-318.

[14] M. ShERESHEVSKY, A complement to Young's theorem on measure dimension: the difference between lower and upper pointwise dimension, Nonlinearity 4 (1991), 15-25.

[15] D. Thompson, The irregular set for maps with the specification property has full topological pressure, Dyn. Syst. 25 (2010), 25-51.

DEPARTAMENTo de MATEMÁtica

INSTITUTO SUPERIOR TÉCNICO

1049-001 LISBOA

PORTUGAL

E-mail address: barreira@math.ist.utl.pt

Departamento de Matemática

INSTITUTO SUPERIOR TÉCNICO

1049-001 LISBOA

PORTUGAL

E-mail address: cvalls@math.ist.utl.pt
SCHOOL OF MATHEMATICS AND STATISTICS MINNAN NORMAL UNIVERSITY

ZHANGZHOU, 363000

P. R. CHINA

E-mail address: li-jinjun@163.com 